\title{
Buchbesprechungen - Book Reviews - Livres Nouveaux
}

International Congress of Gastroentcrology. Fifth Meeting of L'Association des Sociétés Européennes et Méditerranéennes de Gastro-Entérologie. London, July 18-21, 1956. S. Karger, Basel I New York 1957. XX + 63-1 p., zahlreiche Abb. sF . 78.-. (Separatum aus Gastroenterologia, Vol. 86, Nr. 3-5.)

Als erstes Hauptthema werden die nichtbösartigen Erkrankungen des Oesophagus besprochen. Mit Hilfe intraoesophagealer Druckmessungen konn-ten neue Gesichtspunkte über den normalen Ablauf des Schluckaktes gewon-nen werden. Hierbei sinkt der Tonus des Oesophagus an seinem obern und unteren Ende ab, die peristaltische Welle kann sich von oben nach unten gegen den Magen zu ausbreiten. Beim Cardiospasmus fällt die Erschlaffung der pars abdominalis oesophagei aus. Pathologisch-anatomisch konnten in solchen Fallen Degenerationserscheinungen im Auerbach'schen Nervenplexus nachgewie-sen werden. Zur Abgrenzung funktioneller Krankheitsbilder von organischen Läsionen wird die Pharmakoradiographie, vor allem mit Metacholin, emp fohlen.

Weiterhin stehen die Präkanzerosen des Magen-Darm-Traktes zur Dis-kussion: Im Bereiche von Colon und Rectum sind die familiäre Polyposis, die chronische Colitis ulcerosa, die sekundäre Degeneration primär gutartiger Tumoren und die chronische Diverticulitis als Vorzustand der Karzinombildung anzusehen. Unentschieden bleibt die Frage, ob die chronische Gastritis als Vorläufer des Magenkarzinoms zu gelten habe (G. E. Konjetzny, H. H. Berg). Gutmann und Albot halten dies fur ein sehr seltenes Geschehen. Sie schreiben der sekundären Degeneration des Uicus ventriculi eine große Bedeutung bei. Praktisch gesehen ist diese Beobachtung sehr wichtig: Bei alien Patienten mit Ulcus ventriculi, wo auch immer die Nische im Magen lokalisiert sei, ist eine 4- $\beta$ wöchige intensive unspezifische Reizkörpertherapie durchzuführen. Verschwindet unter dieser Behandlung die Ulcusnische nicht, ist mit der karzinomatösen EntaГtung des Ulcus zu rechnen und der Patient dem Chirur-gen zu überweisen.

Unter dem dritten Hauptthema wird die Colitis ulcerosa behandelt. Es findet sich eine eingehende Beschreibung der klinischen Symptomatologie und Pathogenese dieses Krankheitsbildes. Therapeutisch wird der psychiatrischen Betreuung des Patienten großes Gewicht beigemessen. Medikamentös steht zurzeit die Behandlung mit Salicylazosulfapyridin im Vordergrund; die Be-handlungserfolge mit Cortison sind offensichtlich nur von kurzer Dauer. Die Indikation zur operativen Behandlung: Ileostomie und Colectomie wird aus-führlich beschrieben.

Jeder Arzt, der sich für diese Krankheitsbilder interessiert, wird immer wieder mit Gewinn zu dem tadellos ausgestatteten Buch greifen.

H. Stös.sel, Zurich.

Buchbesprechungen - Book Reviews - Livres Nouveaux 187

Lehrbuch der speziellen pathologischen Anatomie, II. Band, 2. Lieferung (Harnorgane).

Begründet von E. Kaufmann und M. Staemmler. Walter de Gruyter \& Co., Berlin 1957. XVI + 405-912 S. 452 Abb. DM 90.20, Subskr.-Pr. DM 82.00. 
Auch die jetzt vorliegende Lieferung ist, wie die übrigen an dieser Stelle referierten, in völlig neuem Gewand erschienen und mil guten Abbildungen ausgestattet worden. Als Verfasser der Lieferung zeichnet Martin Staemmler, ein sehr bekannter Spezialist auf dem Gebiet der Nierenpathologie. Die nach den einzelnen Kapiteln eingeschalteten Literaturverzeichnisse sind ebenso wie der Gesamttext stark modernisiert worden; auch neue und neueste Arbeiten werden in guter Auswahl berücksichtigt. H. U. Zollinger, St. Gallen. W. Bolt, W. Forssmann und H. Rink: Selektive Lungenangiographie in der pracoperativen Diagnostik und in der innercn Klinik. Georg Thieme Ver-lag, Stuttgart 1957. XVI + 200 S., 205 Abb. DM 54.-.

Der Herzkatheterismus scheint für die moderne Herz- und Lungenklinik zur selbstverständlichen Routinemethode geworden zu sein, er befruchtet aber auch das Schrifttum. Im gleichen Verlag erschienen bereits eine Monographie von Bayer, Loogen und Wolter über den «Herzkatheterismus bei angeborenen und erworbenen Herzfehlem» (1954), eine umfangreiche Darstellung der <> Herz-krankheiten im Säuglingsalter» von Rossi (1954), eine Monographie über die «Arteriographie der Lunge und die Kontrastdarstellung der Herzhöhlen am lebenden Menschen» von L. Lòffler (1955) und ein Buch über die «Haemo-dynamik des Herzens im Röntgenbild» von Thurn (1956), alles Bücher, die die Möglichkeiten und Ergebnisse der Herzsondierung darstellen, d. h. ohne die Einführung dieser Melhode nicht geschrieben worden wären. Nun folgt die monographische Beschreibung der von Bolt und Rink propagierten röntgeno-logischen Darstellung der kleinen Lungengefäße bei Lungen- und Herzkrank-heiten. Bei dieser Methode werden 5-10 ccm eines Kontrastmittels durch eine Herzsonde, deren Spitze unter Durchleuchtungskontrolle in das zu unter-suchende Lungenfeld vorgeschoben wird, unter geringem Druck injiziert und eine, eventuell mehrere Röntgenaufnahmen gemacht. Der Anteil des Nobel-preisträgers Forssmann an diesem Buch, das den seinerzeit in Deutschland tonangebenden Lungenklinikern Ludolph Brauer und Ferdinand Sauerbruch gewidmet ist, deren Skepsis und Ablehnung damals Forssmann zur Aufgabe seiner Versuche zwangen, besteht sinnigerweise in einer kurzen historischen Darstellung über die Entwicklung der Herzsondierung mit der Reproduktion einiger Original-Röntgenbilder seiner Selbstversuche mit eingeführter Herzsonde aus den Jahren 1929/30 und einer kinematographischen Darstellung der Kontrastfüllung der Herzhöhlen beim Hund aus der gleichen Zeit.

Bei der Füllung der Lungengefäße unterscheiden die Autoren eine arte-rielle, eine kapilläre und eine venose Phase, die sich durch kontinuierliches Nachspritzen auf einer Röntgenaufnahme simultan darstellen lassen. Die zahl-reichen Abbildungen im speziellen Teil zeigen zur Hauptsache die arterielle Phase. Für die Beurteilung der Funktionstüchtigkeit des Lungengefäßsyslems gelten folgende Kriterien:

188 Buchbesprechungen - Book Reviews - Livres Nouveaux

In hypoventilierten, bzw. nicht mehr ventilierten Bezirken, also zum Bei-spiel auch an ei $\pi$ er teilweise wegen Pneumothorax kollabierten Lunge findet man eine Engerstellung der kleinen Gefäße mit einer zeitlich verlängerten ka-pillären Füllungsphase, wobei es sich um die röntgenologische Darstellung des Einflusses der alveolären Gasspannungen auf den Tonus der kleinen Lungen-gefäße handelt. Eine fehlende kapilläre Füllungsphase ist gleichbedeutend mit einem Verlust an durchgängigen Kapillaren in dem betreffenden Gebiet, z. B. als Folge destruierender oder obstruierender Prozesse im Bereiche der kleinen und kleinsten Gefäße. Dieser Befund mit abrupten Gefäßabbrüchen und Kaliber-schwankungen, ein Gefäßverlust kombiniert mit einem röntgenologisch norma-len Lungenparenchym ist typisch für die primäre 
und sekundäre Pulmonal-sklerose. Werden die Lungengefäße durch destruktive Parenchymveränderun-gen, z. B. Silikose, mit betroffen, so zeigen sich neben Verziehungen und un-regelmäßigen Aufteilungen des Gefäßbaumes die Blockierungen bereits im Bereiche der kleinen Arterien.

Im speziellen Teil des Buches sind zahlreiche Beispiele von selektiven Lungenangiogrammen bei verschiedenen Lungenerkrankungen sowie bei an-geborenen Herzfehlern und bei der Mitralstenose angeführt und zum Teil mit den Befunden zusätzlicher Untersuchungen, wie intrakardiale Druckmessung, Spiromelrie, Blutgasanalyse, Bronchographie und IsotopenThorakographie, er-gänzt. Bemerkenswert ist, wie gelegentlich relativ kleine röntgenologische Lun-genbefunde mit deutlichen Gefäßveränderungen kombiniert sind.

Neben der Klärung grundsätzlicher pathophysiologischer Fragestellungen sehen die Autoren die wichtigste Indikatiou für die selektive Lungenangio-graphie bei Lungen- und Herzkrankheiten im Rahmen der praeoperativen Be-urteilung des Operationsverfahrens - zum Beispiel beim alten Pneumothorax mit Pleuraschwarte, Dekortikation oder Pneumonektomie - und für die Abschälzung des Operationsrisikos und der funktionellen Spätprognose in all den Fallen, bei denen die sonstige Diagnostik nicht ausreicht. Die Methode setzt natürlich den Herzkatheterismus mit seinen allerdings geringen Risiken voraus, der aber nicht allzu selten durch das gar nicht so einfache Sondieren des je-weils gewünschten lobären oder gar segmentären Astes der Art. pulmonalis zeitlich erheblich verlängert wird. Ob die Lungenangiographie als praeopera-tive Untersuchungsmethode größere Bedeutung und Verbreitung finden wird, bleibt abzuwarten. Bisher scheint das noch nicht der Fall zu sein, jedenfalls zitieren die Autoren, was Methode und Ergebnisse betrifft, fast ausschließlich eigene Arbeiten.

Das Ziel einer den Anforderungen des Kranken genügenden und den heu-tigen chirurgischen Möglichkeiten entsprechenden möglichst genauen praeoperativen funktionellen Diagnostik läßt sich, was die Lungenkrankheiten betrifft, in der Mehrzahl der Fälle auch mit einfacheren Methoden und auch ohne Herzkatheterismus erreichen. Der Kenner der einschlägigen Literatur wird wie der Rezensent feststellen, daß im «Arbeitskreis» um Prof. Knipping (Med.

Universitätsklinik, Köln), aus dem vor wenigen Jahren noch Arbeiten über die angebliche Gefährlichkeit und der deshalb den Kranken kaum zumutbaren Arterienpunktion erschienen sind, nun innerhalb von 6 Jahren bereits mehr als 2000 selektive Lungenangiogramme und damit auch Herzsondierungen durchgeführt wurden, was wohl kaum ohne großzügige Indikationsstellung Buchbesprechungen - Book Reviews - Livres Nouveaux 189

für eine nicht ganz harmlose Untersuchung und damit Meinungsänderung über das dem Kranken «Zumutbare» möglich gewesen ist.

Die selektive Lungenangiographie stellt zweifellos eine Bereictterung der röntgenologischen Untersuchungstechnik der Lunge und der Lungengefäße dar, und die MonogГaphie demonstriert zusammenfassend an einem umfang $\Gamma$ eichen und vielseitigen Krankengut eine spezielle Anwendungsmöglichkeit des HerzkatheteГismus. A. Bühlmann, Zurich. O. Marchesani und H. Sautter: Atlas des Augenhintcrgi nudes. Verlag von Urban \& Schwarzenberg, München/Berlin 1956. Lieferungen 11-20. DM30.- je Lieferung. Nachdem nun das Gesamtwerk von Marchesani-Sautter, Atlas des Augen-hintergrundes, vorliegt, lohnt es sich, nochmals etwas eingehender darauf ein-zutreten. Zwei bedeutende Namen sind mit dem Werk verbunden. Marchesani hatte den Atlas begonnen, ein reiches Bildmaterial gesammelt. Die Zerstörung eines Teils der Bilder durch den Krieg hat das Werk ein erstes Mai 
gefährdet. der frühzeitige Tod des großen Ophthalmologen ein zweites Mai. Sautter hat es dann übernommen, den Bau fortzusetzen und zu vollenden. In verhältnis-mäßig kurzer Zeit hat er das Bildmaterial zusammengestellt, ergänzt und von prägnantem Text begleitet. Der heute vorliegende umfangreiche Atlas ehrt seine Verfasser. Marchesani wiirde sich darüber freuen, und Sautter kann des Dankes sicher sein, daß er das Werk in diesem Sinn und Geist abgeschlossen hat. Die didaktische und übersichtliche Zusammenstellung von 262 farbigen Augenhintergrundsbildern mit erklärendem Begleittext ist wohl in erster Linie für den Spezialarzt gedacht, vermag aber auch dem Nichtophthalmologen, im be-sondern dem Internisten, einen nicht nur wertvollen, sondern in mancher Hin-sicht unentbehrlichen Einblick zu verschaffen. Jeder Arzt, der sich die Mühe nimmt, in besondern Fallen bei seinen Patienten auch einen Blick auf den Augenhintergrund zu werfen, wird im Atlas schönstes Vergleichsmaterial fin-den. Es scheint mir deshalb angezeigt, auch in einer nicht ophthalmologischen Zeitschrift einen etwas ausführlichen Überblick über das Werk zu geben.

Im ersten Band fïnden wir: 1. Vergleichende Ophthalmoskopie, nur einige Abbildungen von Augenhintergründen bekannter Tiere. 2. Den nomtalen Augenhintergrund des Menschen als kurze Auffrischung von Reminiszenzen des Stadiums. 3. Phgsiologische Varianten und Mißbildungen, vor allem erstere geben dem Nichtfacharzt wertvolle Hinweise für die Differentialdiagnose normal-pathologisch. 4. Erkrankungen des Sehnerven; die Stauungspapille ist durch sehr gute Abbildungen charakterisiert. 5. Erkrankungen der Netzhaut; die heute so häufigen Gefäßerkrankungen werden anhand sehr zahlreicher Abbildungen ausführlich und anschaulich erörtert. Dieses Kapitel ist für den Nichtfacharzt besonders aufschlußreich. In einem gesonderten Abschnitt werden auch kurz die Erkrankungen des Blutes und der blutbildenden Organe mit den charak-teristischen Fundusveränderungen gezeigt. Wenn es sich dabei auch um sel-tenere Bilder handelt, können sie doch besonders den Internisten interessieren.

Der zweite Band beginnt mit der Fortsetzung der Erkrankungen der Netzhaut; die Degenerationen, die Netzhautablösung und die Tumoren. Dann folgt 6. Erkrankungen der Aderhaut. Auch hier zuerst die Gefäßerkrankungen, im

190 Buchbesprechungen - Book Reviews - Livres Nouveaux

besondern die Aderhautsklerose, ein für den Internisten wichtiger Befund. Dann folgen die Entzündungen, Degenerationen und Tumoren. In einem letzten, 7. Kapitel Folgen von Verletzungen.

Anläßlich der Besprechung der ersten Lieferung batten wir erwähnt, daß das hier in Entstebung begriffene Werk eine besonders schöne Zusammenstellung zu werden verspricht. Der Verlag Urban \& Schwarzenberg hat durch vorzügliche Bildwiedergabe und Ausstattung dazu beigetragen, die Erwartungen zu erfüllen und den Atlas Marchesani-Sauttcr zu einer wirklichen Bereieherung des medizinischen Schrifltums und zu einem eigentlichen Nachschlagewerk, be sonders auch für Nichtfachärzte, zu machen. A. Bangerter, St. Gallen. Verhandlungeii der Deutschen Gescllschaft für Krcislaufforschung. 22. Tagung. 6.-8. April 19Õ6, in Bad Nauheim. 1). Steinkopff, Darmstadt 1956. XXXVIII + 374 Seiten, 140 Abb. in 188 Einzeldarstellungen und 29 Tab. DM 48.-.

Korrelationen zwiscben Herz und Gefäßsystem stellen das Hauptthema dieses Tagungsberichtes dar. Im ersten Teil über die normale und pathologische Physiologie des Kreislaufs legt Reichel (München) eine neue Theorie der Herz-dynamik dar, wonach diese nicht, wie bisher angenommen, vom venösen Zufluß, sondern von muskelphysiologischen Gesetzmäßigkeiten reguliert wird. Wetterer (München) erläutert die Wirkung der Herztätigkeit auf die Dynamik des 
Arteriensystems und Guuer (Bad Nauheim) weist auf die Wechselbeziehun-gen zwischen Herzund Venensystem hin, wobei er vor allem die Bedeutung des «zentralen oder intrathorakalen Blutdepots» für die dynamische Regulation hervorhebt, die über Dehnungsrezeptoren im linken Vorhof erfolgen soil. Durch eine Reihe weiterer Vorträge werden diese physiologischen Referate ergänzt.

Im zweiten Teil zur Klinik der Kreislauferkrankungen gibt Sjöstrand (Stockholm) seine Untersuchungsergebnisse über die Korrelationen verschiedener Kreislaufdaten (Herzvolumen, Gesamt-Blutvolumen, Schlagvolumen, Minutenvolumen, Sauerstoffverbrauch, Pulsfrequenz, a.v. Sauerstoffdifferenz, art. Milchsäurekonzentration usw.) bei Gesunden und Herzkranken wieder. Delius (Baden-Baden) zeigt das Verhalten des Herzmuskels bei primären Störungen des Gefäßsystems und er faßt alle Erkrankungen des linken Ventrikels, die durch eine dauernde Störung des Gefäßsystems hervorgerufen werden, als «adaptive Myocardiopathien» zusammen. Schwiegk (Marburg) berichtet über Auswirkungen von Funktionsstörungen des Herzens auf die Peripherie am Beispiel der Herzinsuffizienz und legt vor allem die Probleme der Regulation des Blutvolumens und des Mechanismus der Oedem-Entstehung unter besonderer Berücksichtigung der Aldosteron-Wirkung dar. Auch diese Hauptreferate wer den durch zahlreiche Einzelvorträge, teils das Hauptthema ergänzend, teils mit freier Themenwahl, umrahmt, auf die hier aber nicht näher eingegangen wer den kann. Der Verhandlungsberícht ergibt somit eine gute Synthese von großem Einzelwissen über die physiologischen und pathologischen Korrelationen zwi schen Herz- und Gefäßsystem. Das Buch dürfte daher sowohl für den Physiologen wie für den Kliniker, der das häufig Rätselhafte im Kreislaufgeschehen zu verstehen sucht, von großem Interesse sein L. Hensle $\tau$, St. Gallen. BuchbespreL·hungen - Book Be views - Livres Notiveaux 191 D. Woolfolk Barrow: The Clinical Management of Varicose Veins. Second Edition. Paul B. Hoeber, Inc., New York 1957. XIII + 169 p., 70 fig. \$6.90.

Einleitend bespricht der Autor die wichtigsten Fragen aus der Anatomic und Physiologie der Venen, der Pathogenese und Histologie der Varicen an den unteren Extremitäten. Anschließend geht er näher auf die Untersuchungs-methodik ein. Hier wird besonders ein modifizierter Versuch nach Trendelcn-burg dargestellt, durch den die Entscheidung möglich sei, ob eine variköse Bildung durch eine Insuffizienz im BeГeich der Vena saphena magna, der V. saphena parva oder der oberflächliche und tiefe Venen verbindenden Vv. com-niunicantes hervorgerufen wird. Die Therapie ist vor allem eine chirurgische mit den üblichen, übersichtlich und anschaulich erläuterten Maßnahmen: Unterbindung, Exstirpation mit der Sonde, Exzision größerer Varixknäuel. Das Vorgehen muß modiñziert werden, je nachdem welches der Venengebiete in-suffizient ist. Die Injektionsbehandlung wird als ergänzende Maßnahme oder in Fallen beginnender Varicosis sehr empfohlen, Technik, Indikationen und Kontraindikationen werden recht ausführlich dargelegt. Schließlich werden noch die Komplikationen abgehandelt: Hämorrhagie, subcutane Ekchymosen, Erysipel, Cellulitis, Ekzem, UIcus und Thrombophlebitis. Bei der Ulcustherapie steht die Varicenbehandlung ganz im Vordergrund. Besonders sind hier in-suffiziente, die Ulcusbildung unterhaltende Vv. communicantes («feeder veins») auszuschalten. Als Standardtherapie der oberflächlichen Thrombophlebitis wird die hohe Unterbindung der V. saphena magna durchgeführt, eine Antikoagu-lantienbehandhmg nur bei Verdacht auf das Vorliegen einer tiefen Thrombophlebitis eingeleitet. 
Das Buch ist sehr empfehlenswert: es ist klar geschrieben, in der Anord-nung übersichtlich. Der Text wird ergänzt durch gute Schemata und Photo-graphien sowie durch ein ausführliches

Literatur- und Sachwortverzeichnis.

P. Schmuziger, St. Gallen.

R. Heinecker: EKG-Fibel. Geleitwort von F. Hoff. 2., verbesserte Aufl. Verlag G. Thieme, Stuttgart 1957. XII + 200 S. 182 Abb. in 308 Einzeldarstellungen und 34 schematische

Darstellungen. DM 19.80.

Das rasche Erscheinen der 2. Auflage zeigt, daß die EKG-Fibel von Hei necker von vielen Studierenden und Ärzten günstig aufgenommen wurde. Die 2. Auflage ist nur unwesentlich verändert. Es wird auch diese Neuauflage zweifellos sehr geschätzt werden. R. Hegglin, Zurich.

Paul D. White: Die Schlüssel zur Diagnose und Therapie der Herzkrankheiten. Verlag Dr. Dietrich Steinkopff, Darmstadt 1957. VIII + 200 S., 40 Abb. DM 19.50.

Das Buch des bekannten amerikanischen Kardiologen White: «Clues in the Diagnosis and Treatment of Heart Disease» liegt nun in deutscher Übersetzung vor. Das Buch wird sich auch im deutschen Sprachgebiet, besonders bei praktischen Ärzten, viele Freunde erwerben, weil es in echtem klinischem Geist geschrieben ist und hilft, die Diagnose der Herzkrankheiten aus der Anamnese und einfachen Untersuchungsbefunden zu stellen. Bewußt wird auf die Bewertung der komplizierten Untersuchungsmethoden verzichtet. Das Buch kann alien Ärzten bestens empfohlen werden. R. Hegglin, Zurich.

192 Buchbesprechungen - Book Reviews - Livres Nouveaux

L. Laszt, R. Meier et Aloys Miiller: Comptes Rendus du lie Congrès International d'Angéiologie, Fribomg (Suisse), septcmbre 1955. Editions Uni-versitaires, Fribourg 1956. 755 p., nombreuses fig.

Das Buch, welches die Rapporte und Mitteilungen sowie die Demonstra-tionen des 2.

Internationalen Kongresses für Gefäßerkrankungen in Freiburg (Schweiz) aus dem Jahre 1955 in sich vereinigt, gibt einen sehr umfassenden überblick über die gegenwärtigen Kenntnisse auf diesem immer wichtigeren Gebiet. Die elwa 120 Vorträge eignen sich nicht zur Rezension und müssen im Original nachgelesen werden. Das Buch ist aber in seiner Reichhaltigkeit alien Ärzten, welche sich mit Gefäßerkrankungen beschäftigen und vor allera denen, welche auf diesem Gebiet Forschungsarbeit treiben, sehr zu empfehlen.

R. Hegglin, Zurich.

Varia

The International Society of Cardiology Foundation.

In January 1957 there was established the International Society of Cardiology Foundation in Chicago for the purpose of supporting research in the cardiovascular field anywhere in the world. Requests for such support can be sent to the Board of Directors or to the Advisory Committee of the Foundation as listed below or to the office of the Foundation in Chicago, c/o Dr. Louis Katz, Michael Reese Hospital. A member receiving such application should forward the information to the President, Dr. Paul D. White, who will then pass the recommendation on for processing to other members of the Advisory Committee. The recommendations of the Committee transmitted to the Board of Directors will permit the latter to act.

The Foundation is in a position to receive funds from any part of the world for the purpose for which it was established. In its beginning the available funds are quite limited, but it is expected 
that they will increase with the knowledge that such a source of support of international cardiovascular research exists.

Board of Directors. President: Dr. Paul D. White, Boston (Mass.). Vice Presidents: Dr. John Palmer, Montreal (Canada); Dr. Ignacio Chavez, Mexico City (Mexico); Dr. D.Evan Bedford, London (England). Treasurer: Dr. Louis N. Katz, Chicago (111.). Secretary: Dr. Pierre W. Duchosal, Geneva (Switzerland) . Assistant Secretary: Mrs. Margaret Stern, Michael Reese Hospital, Chicago (111.). Additional Member: Mr. Frank D. Mayer, Chicago (111.). Advisory Committee: Dr. Paul D. White, Chairman, Boston (Mass.); Dr. Ignacio Chavez, Mexico City (Mexico); Dr. Jean Lenègre, Paris (France); Dr. E. Braun-Menendez, Buenos Aires (Argentina); Dr. Pierre Duchosal, Geneva (Switzerland); Dr. Paul Wood, London (England); Dr. Mariano Alimurung, Manila (P.I.). 